\title{
Spiral waves in excitable media with negative restitution
}

\author{
C. W. Zemlin \\ Innovationskolleg Theoretische Biologie, Invalidenstrasse 43, 10115 Berlin, Germany
}

A. V. Panfilov

Department of Theoretical Biology, Utrecht University, Padualaan 8, 3584 CH Utrecht, The Netherlands

(Received 20 December 1999; revised manuscript received 21 June 2000; published 29 March 2001)

\begin{abstract}
We study numerically the dynamics of spiral waves in an excitable medium with negative restitution. For our study we use two models of the excitable medium: a cellular automaton and a reaction-diffusion model. There are no significant effects of negative restitution as long as the slope of the restitution curve is less steep than -1 . In media with slopes steeper than -1 , the dynamics of spiral waves can change significantly: (1) the average restitution time jumps to a value where the slope of the restitution curve is about -1 ; (2) spiral waves can break up into turbulent patterns. We discuss a possible connection between such instabilities and fibrillation in atrial tissue.
\end{abstract}

DOI: 10.1103/PhysRevE.63.041912

PACS number(s): 87.18.-h, 82.20.Wt, 82.40.Ck

Rotating spiral waves occur in a wide variety of nonlinear excitable media [1]. While spiral waves may be stable under certain conditions, they can also break up into turbulent patterns. In the case of the heart, spiral waves cause tachycardia, a dangerous cardiac arrhythmia associated with a rapid heart beat $[2,3]$. Tachycardia can deteriorate into fibrillation, which is fatal if it occurs in the ventricles of the heart, or a serious complication if it occurs in the atria. One possible mechanism of such deterioration is spiral breakup [4-10].

It has been shown that spiral breakup heavily depends on a property of the excitable medium called its restitution curve [11]. The restitution curve of a medium is the dependency of some pulse characteristic on the restitution time, which is the interval between the start of a pulse and the end of the previous pulse. Often the measured characteristic of the pulse is its duration (called action potential duration or APD in the case of cardiac tissue), which yields the APD restitution curve. Another important pulse characteristic is its refractory period (RP), which is the time interval during which a cell cannot be excited after a previous excitation; this leads to the RP restitution curve. In cardiac tissue, the RP and APD restitution curves are similar. Under normal conditions, longer restitution times lead to longer pulse durations and refractory periods, so that the restitution curve has positive slope everywhere. In this case, it has been shown that instabilities, e.g., spiral breakup, can occur if the slope of the restitution curve is steeper than 1 [11].

Experiments show that the slope of the RP restitution curve of cardiac tissue can become negative (we call this negative restitution) [12]. In the case of atrial tissue, negative restitution has been observed in healthy animals, and it became even more pronounced under pathological conditions (chronic atrial fibrillation) [14]. It was also shown in a modeling study that even a small degree of negative restitution can be important for wave propagation in a ring of excitable tissue [13]. In spite of the existence of negative restitution in cardiac tissue, the influence of negative restitution on spiral wave dynamics has not yet been studied.

In this paper, we study numerically how negative restitution can affect spiral wave dynamics. We are interested in general mechanisms; therefore, we look at restitution curves that are simple rather than realistic for heart tissue.

We developed two types of model of an excitable medium with negative restitution: a cellular automaton (CA) model and a reaction-diffusion (RD) type model. Both types of description of excitable media-CA [5] and RD [4,6-8] -have been used successfully to study spiral breakup in media with positive restitution.

CA model. We modified a cellular automation model [15] in a way that allows us to input the restitution curve directly. In our model, each cell can be in one of the following three states: resting, excited, or refractory. If a cell is in the rest state, we check at every time step whether one of its eight closest neighbors is in the excited state. If so, we increase an inner variable called the excitation counter by 1 for each excited neighbor at each time step. If the excitation counter exceeds a predefined threshold $(h)$, the cell switches to the excited state. In the excited state, a cell can excite its neighbors for a constant number of time steps $\left(t_{\mathrm{ex}}\right)$ and then switches to the refractory state, where it again stays for a constant number of time steps. At the end of the refractory state, the cell switches back into the rest state. The "action potential" in the CA model is therefore fully characterized by the times at which the cell is excited and at which it returns to the rest state, and the shape of the action potential can be considered rectangular.

Now we make the restitution curve of the CA model nonconstant. To achieve that, we need the duration of the refractory period to depend on the time spent in the rest state before excitation. In the CA model, the refractory period $\left(t_{\text {ref }}\right)$ is the sum of the times spent in the excited and refractory states. So by letting the duration of the refractory state depend on the restitution time, an arbitrary restitution curve can be forced upon the model. In our simulations, we always used $t_{\mathrm{ex}}=10$ and $h=19$.

$R D$ model. We also used a reaction-diffusion model of FitzHugh-Nagumo type piecewise linear "Pushchino kinetics" [16]:

$$
\frac{\partial e}{\partial t}=\frac{\partial^{2} e}{\partial x^{2}}+\frac{\partial^{2} e}{\partial y^{2}}-f(e)-g,
$$



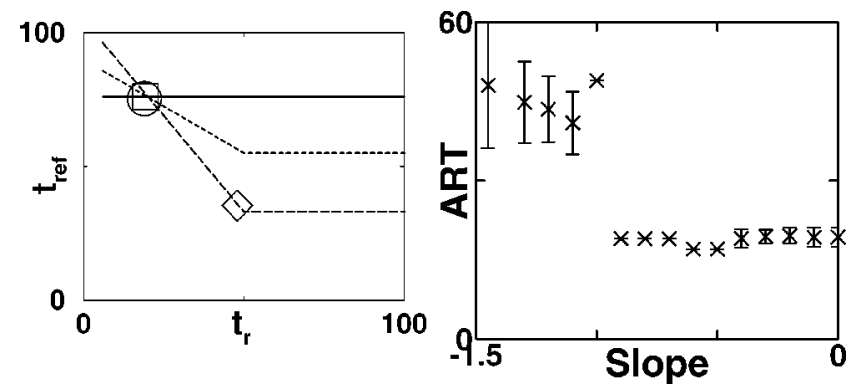

FIG. 1. Left: Restitution curves used in our CA model. $t_{\text {ref }}\left(t_{r}\right)=76+\left(t_{r}-20\right) \times$ slope $\left(\right.$ for $\left.t_{r}<50\right)$ and $t_{\text {ref }}\left(t_{r}\right)=76+30$ $\times$ slope (for $t_{r} \geqslant 50$ ). The restitution curves shown have slope 0 (solid), -0.7 (dashed), and -1.45 (long dashed). Spiral locations are indicated by a square (for slope 0 ), a circle (slope -0.7 ), and a diamond (slope -1.45 ). Right: The range of average restitution times (ARTs) vs slope. The error bars show the standard deviations, but they are smaller than the symbol size for slopes less steep than -1 . Medium size was $40 \times 40$.

$$
\frac{\partial g}{\partial t}=\varepsilon(e, g)(e-g)
$$

where $e$ is the transmembrane potential and $g$ is the gate variable. In order to make the shape of the action potential as simple as possible, we used the following nonlinear function $f(e)$ [17]: $f(e)=\lim _{c \rightarrow \infty} C e$ when $e<0, f(e)=(e-0.1)$ if $0 \leqslant e \leqslant 1$, and $f(e)=\lim _{C \rightarrow \infty} C(e-1)$ when $e>1$. For this shape of $f(e)$, the excitation pulse has a plateau region at $e=1$, the rest state at $e=0$, and the shape of the pulse is close to rectangular. To model the regions of infinite slope of function $f(e)$ numerically we use the IF operators stating that if the variable $e$ is above 1 it is set to 1 , and if $e<0$, it is set to 0 . It has been shown that this procedure gives a sufficiently precise solution of Eq. (1) with $f$ as given above [18]. In our basic model, $\varepsilon(e, g)=0.067$ if $0.1<e<0.99$ and $\varepsilon(e, g)=0.1$ for other values of $e$. Such a model has an almost flat restitution curve (the solid line in Fig. 1). In order to describe a medium with negative restitution, we made the function $\varepsilon(e, g)$ dependent on the restitution time. We put $\varepsilon(e, g)=T^{-1}\left(t_{r}\right)$ (if $0.45 \leqslant g \leqslant 0.55$ and $e<0.1$ ), where $t_{r}$ is the restitution time, and $T_{\mathrm{RD}}\left(t_{r}\right)$ is the function using which we set up the desired restitution curve. In fact, if $0.45 \leqslant g$ $\leqslant 0.55, e<0.1$, the excitable medium is in the refractory state and the time interval during which the variable $g$ decreases from $g=0.55$ to $g=0.45$, is given by $T_{\mathrm{RD}}\left(t_{r}\right) \ln 0.55 / 0.45$. Therefore, by choosing the function $T_{\mathrm{RD}}\left(t_{r}\right)$, we can construct an excitable medium with any desired dependence of the refractory period on $t_{r}$.

For numerical modeling of Eq. (1), we used the explicit Euler method with Neumann boundary conditions and a rectangular grid. Numerical integration was performed with a space step $h_{s}=0.6$ and a time step $h_{t}=0.03$. The error in these computations, estimated using the difference between the computed and the analytically found velocity of plane wave propagation [17], was about $5 \%$.

To initiate the spiral wave we used in both models initial data corresponding to a two-dimensional (2D) broken wave front, or alternatively the $S_{1} S_{2}$ stimulation protocol, which is often used in experimental electrophysiology [19]. Note that we chose the parameters of the CA model such that the spirals closely match those in the RD model. We characterize spirals by their average restitution time (ART). To find the ART we first compute the restitution times at each point of the excitable medium and find their spatial average. The restitution times were evaluated as the time the point spent in the rest state prior to the preceding excitation. The rest state in the RD model was determined as the time between the end of the refractory period and the next excitation. The refractory period was estimated to be the time interval from the beginning of excitation until the moment of time when the variable $g$ reaches the value $g=0.45$ at the wave back.

We start with a simple analysis. Consider stimulation of an excitable medium with a constant period $T$ and denote the refractory periods of the successive pulses as $\left(t_{\text {ref }}\right)_{n}$ and their restitution times as $t_{r, n}$. Because the period of stimulation is constant, $\left(t_{\text {ref }}\right)_{n}+t_{r, n}=T$. The refractory period of the pulse $t_{\text {ref }, n+1}$ is determined by the previous restitution time, or $t_{\text {ref }, n+1}=f\left(t_{r, n}\right)=f\left(T-t_{\text {ref }, n}\right)$, where $f$ is the restitution curve, and the dynamics are given by iteration of this discrete map. A fixed point of this map corresponds to a train of pulses of equal length. Such a fixed point is unstable whenever $\left|d f / d t_{r}\right|>1$ [11]. The case $d f / d t_{r}>1$ corresponds to the positive restitution studied earlier, and $d f / d t_{r}<-1$ corresponds to negative restitution. Therefore, we expect instabilities for negative restitution if the slope becomes steeper than -1 .

In order to study the effect of negative restitution on spiral wave dynamics in our CA model, we first induced a spiral wave in a medium with a constant restitution curve. For our CA model parameters, the ART is 20; this value is marked on the restitution curve (square in Fig. 1, left panel). Then we generated a family of restitution curves that all go through this location $\left(t_{r}=20, t_{\text {ref }}=76\right)$ but have slopes between 0 and -1.5 . These restitution curves are linear up to $t_{r}=50$ time units and constant for $t_{r}>50$ time units. Three examples of such restitution curves together with the computed location of spirals are shown in Fig. 1, left panel. Note that, because all restitution curves go through the point $t_{r}$ $=20, t_{\text {ref }}=76$, there exists a spiral wave solution with $t_{r}$ $=20, t_{\text {ref }}=76$, but it does not mean that this solution is stable. From Fig. 1, left panel, we see that for slopes 0 and -0.7 the spiral location is close to the spiral location for a constant restitution curve, but for slope -1.45 it is considerably shifted to the right. The complete dependency of the spiral location on the slope is shown in Fig. 1, right panel. We see that for slopes less steep than -1 the ART remains almost the same as for the constant restitution curve, with only small standard deviations in the ART. For slopes steeper than -1 , the ART jumps to much higher values. The ART is then close to 50 , the value at which the oblique segment of the restitution curve ends. Also, for slopes steeper than -1 , the ART has a larger standard deviation. This reflects the nonstationarity of spirals that we observe for such restitution curves. For slopes steeper than -1.15 we frequently observed breakup, which was more pronounced for still steeper slopes. In some cases, the spirals disappeared at the boundary. 

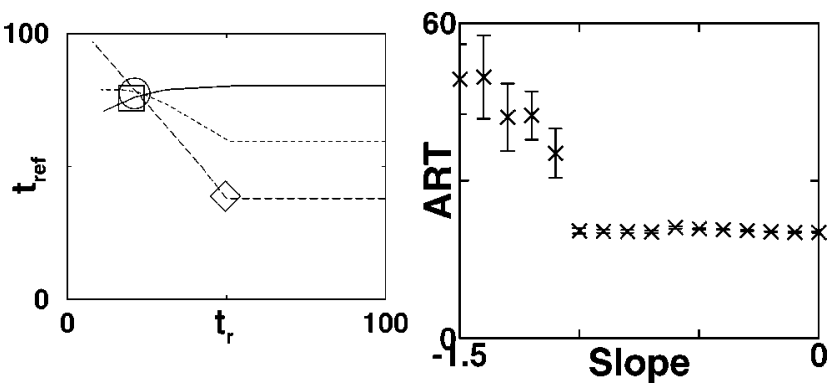

FIG. 2. Restitution curves and corresponding range of average restitution times in the RD model. Restitution curves were set up using $T_{\mathrm{RD}}\left(t_{r}\right)=300+5\left(t_{r}-20\right) \times$ slope (for $\left.t_{r}<50\right)$ and $T_{\mathrm{RD}}=300$ $+150 \times$ slope (for $t_{r} \geqslant 50$ ). Medium size was $300 \times 300$; the meaning of the symbols is the same as in Fig. 1.

We did similar computations in the RD model. By choosing functions $T_{\mathrm{RD}}\left(t_{r}\right)$, we generated a family of restitution curves similar to those used in the CA model (see Fig. 2, left panel). We see that as for the CA model the ART of a spiral wave is almost unchanged for the restitution curves with slopes 0 and -0.7 and it is shifted to the right for slope -1.4 . We also see that the dependency of the ART on the slope in the RD model (Fig. 2, right panel) is similar to that in the CA model, but there are some differences: First, the rise of the ART in the RD model is less abrupt; second, the breakup of spirals for slopes steeper than -1 is not as pronounced as in the CA model.

In our view, this shift of ART can be explained by the following simple consideration. It is reasonable to assume that spiral waves tend to minimize their period. Usually, this results in the selection of the smallest possible distance between the wave front and wave back in a spiral. In physiology such a distance is called the excitable gap; in our case it is just the restitution time. However, for media with negative restitution, the minimal excitable gap (restitution time) does not necessarily mean the minimal period. Because the period of a spiral wave is $T=\left(t_{\text {ref }}+t_{r}\right)$, the minimum is reached at $d T / d t_{r}=0, d^{2} T / d t_{r}^{2}>0$, which implies $d t_{\text {ref }} / d t_{r}=-1$, $d^{2} t_{\text {ref }} / d t_{r}^{2}>0$. So, according to the period minimization principle, for a restitution curve with second derivative everywhere positive, the spiral should choose an ART for which the slope of the restitution curve is -1 .

Although the results shown in Figs. 1 and 2 are consistent with this explanation, they are not conclusive because the slope jumps discontinuously and takes on only two values for each restitution curve. Therefore, we studied a medium whose restitution curve has continuously varying slope. For both $\mathrm{CA}$ and $\mathrm{RD}$ models, we generated a restitution curve that is constant for $t_{r}>T R_{0}$ and a parabola for $t_{r}<T R_{0}$, such that the slope is -1 at $t_{r}=38.2$. We initiated a spiral wave in such a medium and found its ART (Fig. 3, left panel). We see that the spiral indeed chooses an ART close to the point where the restitution curve has slope -1 (arrow) in both models. We also performed a series of computations in which we shifted the parabola. The results are presented in Fig. 3, right panel; the straight line shows the value of ART at which the restitution curve has slope -1 . We see that for both models the time average of the ART is always in the
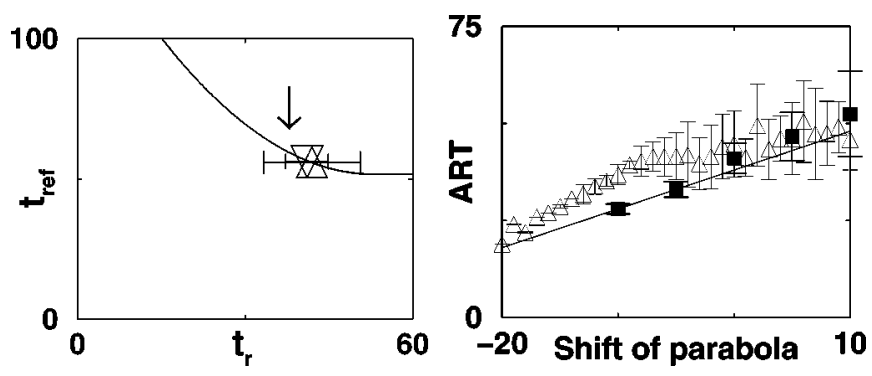

FIG. 3. Spiral in a medium with a parabolic restitution curve. Left: $\mathrm{RD}$ and CA restitution curves are very similar. For better readability, we show only CA restitution curve: $t_{\text {ref }}=\left(T R_{0}\right.$ $\left.-t_{r}\right)^{2} / 30+51.7$ for $t_{r}<T R_{0}$ and $t_{\text {ref }}=51.7$ for $t_{r} \geqslant T R_{0}$; RD restitution curve is $T_{\mathrm{RD}}=\left(T R_{0}-t_{r}\right)^{2} / 6+150$ for $t_{r}<T R_{0}$ and $T_{\mathrm{RD}}$ $=150$ for $t_{r} \geqslant T R_{0}$ with $T R_{0}=53.2$. The arrow shows where the restitution curve has slope -1 . The time averages of the (spatial) average restitution times are indicated by an upward triangle (CA) and a downward triangle (RD). Error bars indicate standard deviations; the large error belongs to the CA model. Medium size was $80 \times 80$ (CA) and $300 \times 300(\mathrm{RD})$. Right: Average restitution time as a function of parabola shift. The expression $T R_{0}$ in the restitution curve definitions is replaced by $T R_{0}+$ (shift of parabola). The corresponding average restitution times are represented by triangles for the CA model and by squares for the RD model. Error bars indicate standard deviations in time. The solid line shows which ART corresponds to slope -1 in the restitution curve.

region where the slope is less steep than -1 (above the solid line). In the RD model, the average ART is almost exactly at slope -1 ; in the CA model, there are some deviations toward larger ARTs. Therefore, we conclude that spiral waves tend to avoid the regions where the restitution curves have slopes steeper than -1 by choosing an ART value at which the slope of the restitution curve is close to and less steep than -1 .

Another important phenomenon that may occur in media with negative restitution is spiral breakup into a complex turbulent pattern. Figure 4 shows examples of such patterns which occur in CA and RD models with a parabolic restitu-
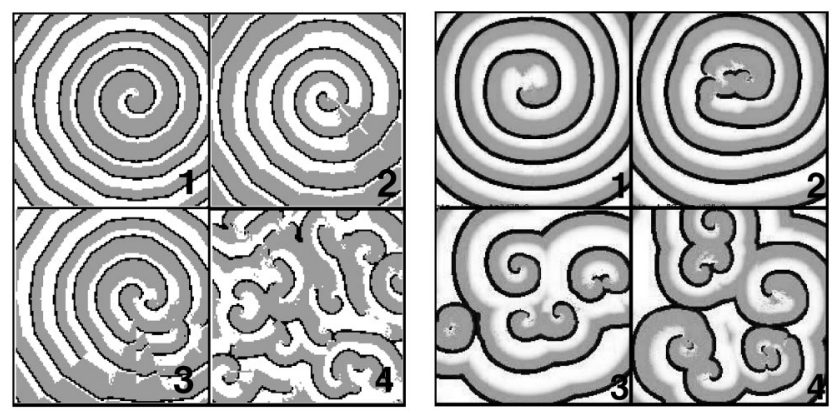

FIG. 4. Spiral breakup for negative restitution. Left: in the CA model, with restitution curve from Fig. 3, left panel, after 97, 101, 110 , and 475 spiral periods (medium size $160 \times 160$ ). We show only the central $120 \times 120$ points of the medium to have the same scaling as in the right image. Right: in the RD model, with the restitution curve $T_{\mathrm{RD}}=\left(53.2-t_{r}\right)^{2} / 3+150$ for $t_{r}<53.2$ and $T_{\mathrm{RD}}$ $=150$ for $t_{r} \geqslant 53.2$, after $22,43,145$, and 211 spiral periods (medium size $721 \times 721)$. 
tion curve. In the CA model the breakup typically occurs in the following way. At some region of the spiral, the excitable gap becomes rather small, leading to a longer duration of the next excitation (due to the negative restitution). This leads to progressively smaller excitable gaps (which can be seen in Fig. 4, left panel, first subfigure). Finally, the wave front breaks; in the case of Fig. 4, left panel, this occurs close to the core of the spiral wave. The open ends of the broken front form new spirals, which in turn break up further, resulting in the patterns presented in Fig. 4, left panel.

In the RD model, the effects are similar (see Fig. 4, right panel); however, the regions of instability are not as regular as in the case of the CA model. The breakup again occurs close to the core of the spiral.

Note that the breakup in the RD model is in general substantially less pronounced than in the CA model. In fact, we have observed a very limited breakup for the parabolic restitution curve from Fig. 3, left panel, and in order to generate a picture comparable to the CA model we increased the curvature of the parabola by a factor of 2 .

The breakup that we observed in media with negative restitution is in some ways less pronounced than the breakup known in media with positive restitution. The main difference is that in media with negative restitution breakup takes quite a long time to develop (tens of rotations), compared to just a few rotations in the case of positive restitution curves [7]. This may be a consequence of the effect found in this paper: shifting of the spiral ART to the region where the slope of the restitution curve is -1 . In fact, the slope -1 is the value at which instability first occurs and therefore the growth rate of the instabilities is minimal, which may explain their slow development. Quite differently, in media with positive restitution, the shift of the ART is absent and the spiral is usually located in the region with slope much steeper than 1 [20], which results in faster growth of instabilities and faster breakup of spirals.

Still, the effect of ART shifting to the point where the slope equals -1 might in certain situations promote spiral breakup. In fact, this shift should be observed not only for restitution curves with negative slope everywhere, but also for restitution curves whose slope is negative just in a small interval. In such a case, the shift can potentially move the spiral from the region where breakup is absent to the region of slope -1 where breakup is possible.

Although the relation between negative restitution and atrial fibrillation is not yet clear, experimental results indicate fibrillation is more easily inducible in atrial tissue with strongly negative restitution [14]. Our results on the instability of spiral waves in media with negative restitution suggest an explanation for how negative restitution can facilitate the development of atrial fibrillation. Note, however, that the restitution curves measured in [14] did not have slopes steeper than -1 .

In this paper, we used general models of excitable media with negative restitution. Therefore, it is likely that the instabilities we found can occur not only in cardiac tissue, but also in the BZ reaction [21,22], neuronal tissue [23], or other excitable media that exhibit negative restitution.
[1] A. T. Winfree and S. H. Strogatz, Nature (London) 311, 611 (1984).

[2] M. A. Allessie, F. I. M. Bonke, and F. J. G. Schopman, Circ. Res. 33, 54 (1973).

[3] J. M. Davidenko et al., Nature (London) 355, 349 (1991).

[4] A. V. Panfilov and A. V. Holden, Phys. Lett. A 147, 463 (1990).

[5] H. Ito and L. Glass, Phys. Rev. Lett. 66, 671 (1991).

[6] M. Courtemanche and A. T. Winfree, Int. J. Bifurcation Chaos Appl. Sci. Eng. 1, 431 (1991).

[7] A. V. Panfilov and P. Hogeweg, Phys. Lett. A 176, 295 (1993).

[8] A. Karma, Phys. Rev. Lett. 71, 1103 (1993).

[9] Z. Qu, J. Weiss, and A. Garfinkel, Am. J. Physiol. 276, H269 (1999).

[10] M. Riccio, M. Koller, and R. Gilmour, Jr., Circ. Res. 84, 955 (1999).

[11] M. Courtemanche, L. Glass, and J. P. Keener, Phys. Rev. Lett. 70, 2182 (1993).
[12] M. Franz et al., Circ. Res. 53, 815 (1983).

[13] Z. Qu, J. N. Weiss, and A. Garfinkel, Phys. Rev. Lett. 78, 1387 (1997).

[14] M. Wijffels, C. Kirchhof, R. Dorland, and M. A. Allessie, Circulation 92, 1954 (1995).

[15] V. S. Zykov and A. S. Mikhailov, Dokl. Akad. Nauk (SSSR) 286, 341 (1986), [Sov. Phys. Dokl. 31, 51 (1986)].

[16] A. V. Panfilov and A. M. Pertsov, Dokl. Akad. Nauk (SSSR) 274, 1500 (1984).

[17] R. Khramov, Biofizika 23, 871 (1978) (in Russian).

[18] A. V. Panfilov and J. P. Keener, SIAM (Soc. Ind. Appl. Math.) J. Appl. Math. 55, 205 (1995).

[19] P. S. Chen et al., Circ. Res. 62, 1191 (1988).

[20] A. V. Panfilov, Chaos 8, 57 (1998).

[21] A. N. Zaikin and A. M. Zhabotinsky, Nature (London) 225, 535 (1970).

[22] A. T. Winfree, Science 175, 634 (1972).

[23] N. A. Gorelova and J. J. Bures, J. Neurobiol. 14, 353 (1983). 